\title{
Lizards along an agricultural land abandonment gradient in Pindos Mountains, Greece
}

\author{
Sylvia Zakkak ${ }^{1}$, John Maxwell Halley ${ }^{2}$, Triantafyllos Akriotis ${ }^{3}$, Vasiliki Kati ${ }^{1}$ \\ 1 - University of Patras, Department of Environmental and Natural Resources Management, Seferi 2, 30100 \\ Agrinio, Greece \\ 2 - University of Ioannina, Department of Biological Applications and Technology, 45110 Ioannina, Greece \\ 3 - University of the Aegean, Department of Environmental Studies, 81100 Mytilene, Greece
}

\section{Supplementary tables}

Table S1. Number of individuals per lizard species recorded in Pindos Mountains area during line transect sampling in spring-summer 2011-2012.

\begin{tabular}{cccccc}
\hline FE class & $\begin{array}{c}\text { Argyriodes } \\
\text { nigropunctatus }\end{array}$ & $\begin{array}{c}\text { Lacerta } \\
\text { viridis/trilineata }\end{array}$ & $\begin{array}{c}\text { Podarcis } \\
\text { muralis }\end{array}$ & $\begin{array}{c}\text { Podarcis } \\
\text { tauricus }\end{array}$ & $\begin{array}{c}\text { Unidentified } \\
\text { individuals }\end{array}$ \\
\hline 1 & & 1 & 30 & & 4 \\
2 & 2 & 1 & 29 & 1 & 2 \\
3 & & 4 & 31 & 1 & 9 \\
4 & 1 & 2 & 34 & & 5 \\
\hline Total & 3 & 8 & 124 & 2 & 20 \\
\hline
\end{tabular}

Forest encroachment, FE class 1: 0-25\% WVC, FE class 2: 25-50 \% WVC, FE class 3: 50-75\% WVC, FE class 4: 75-100 \% WVC, WVC: woody vegetation cover. 
Table S2. Number of individuals per sampling site, recorded in Pindos Mountains area during line transect sampling in spring-summer 20112012.

\begin{tabular}{|c|c|c|c|c|c|c|c|c|c|c|c|c|c|c|c|c|c|c|c|c|c|}
\hline \multirow{2}{*}{ Species } & \multicolumn{20}{|c|}{ Sampling site code } & \multirow{2}{*}{ Tota } \\
\hline & 01 & 02 & 03 & 04 & 05 & 06 & 07 & 08 & 09 & 10 & 11 & 12 & 13 & 14 & 15 & 16 & 17 & 18 & 19 & 20 & \\
\hline A.nigropunctatus & & & & & & & & & & 2 & & 1 & & & & & & & & & 3 \\
\hline L.viridis/trilineata & 1 & 1 & & 1 & & 1 & & 2 & & & 2 & & & & & & & & & & 8 \\
\hline P.muralis & 14 & 1 & 23 & 3 & 3 & 1 & 1 & 11 & 1 & 1 & 4 & 12 & & 3 & 2 & 7 & 1 & 14 & 16 & 6 & 124 \\
\hline P.tauricus & 1 & & 1 & & & & & & & & & & & & & & & & & & 2 \\
\hline Unidentified & 2 & & 1 & 3 & & & & 4 & & & 3 & & & 1 & & 4 & & 2 & & & 20 \\
\hline Total & 18 & 2 & 25 & 7 & 3 & 2 & 1 & 17 & 1 & 3 & 9 & 13 & 0 & 4 & 2 & 11 & 1 & 16 & 16 & 6 & 157 \\
\hline
\end{tabular}


Table S3. Environmental variable values of each sampling site, in Pindos Mountains area [LSU: Livestock Unit as defined by the European Commission (2013)].

\begin{tabular}{|c|c|c|c|c|c|c|c|c|c|c|c|}
\hline \multirow[t]{2}{*}{ Site } & \multirow[t]{2}{*}{$\begin{array}{c}\text { Woody } \\
\text { vegetation } \\
\text { cover }(\%)\end{array}$} & \multirow[t]{2}{*}{$\begin{array}{c}\text { Grazing } \\
\text { intensity } \\
\left(\text { LSU } / \mathrm{km}^{2}\right)\end{array}$} & \multirow[t]{2}{*}{$\begin{array}{l}\text { Average } \\
\text { elevation } \\
\text { (m) }\end{array}$} & \multirow[t]{2}{*}{$\begin{array}{l}\text { Longitude } \\
\text { (WGS 84) }\end{array}$} & \multirow[t]{2}{*}{$\begin{array}{l}\text { Latitude } \\
\text { (WGS 84) }\end{array}$} & \multirow[t]{2}{*}{$\begin{array}{c}\text { Topographic } \\
\text { Wetness Index }\end{array}$} & \multicolumn{3}{|c|}{ Main substrate type (\%) } & \multicolumn{2}{|c|}{$\begin{array}{c}\text { Woody vegetation } \\
\text { cover at microhabitat } \\
\text { scale }(\%)\end{array}$} \\
\hline & & & & & & & $\begin{array}{c}\text { dry } \\
\text { leaves }\end{array}$ & $\begin{array}{l}\text { bare } \\
\text { ground }\end{array}$ & grass & $\begin{array}{l}\text { less than } \\
3 \mathrm{~m} \text { tall }\end{array}$ & $\begin{array}{c}\text { more than } \\
3 \mathrm{~m} \text { tall }\end{array}$ \\
\hline 01 & $50-75$ & 7.2 & 760 & 21.34 & 40.18 & 24.57 & 0.33 & 0.08 & 0.58 & 35.00 & 14.17 \\
\hline 02 & $0-25$ & 7.0 & 790 & 21.34 & 39.79 & 25.08 & 0.25 & 0.42 & 0.33 & 11.67 & 7.50 \\
\hline 03 & $25-50$ & 8.0 & 870 & 21.19 & 40.20 & 24.85 & 0.09 & 0.45 & 0.42 & 0.00 & 0.00 \\
\hline 04 & $50-75$ & 1.8 & 950 & 21.23 & 40.15 & 24.68 & 0.08 & 0.33 & 0.58 & 2.92 & 2.50 \\
\hline 05 & $75-100$ & $\overline{6.0}$ & 940 & 20.96 & 40.20 & 24.33 & 0.67 & 0.33 & 0.00 & 5.42 & 19.17 \\
\hline 06 & $25-50$ & 9.2 & 1000 & 20.92 & 40.23 & 24.73 & 0.64 & 0.27 & 0.09 & 17.27 & 17.27 \\
\hline 07 & $25-50$ & 5.5 & 870 & 20.89 & 40.27 & 23.83 & 0.17 & 0.42 & 0.42 & 6.67 & 12.50 \\
\hline 08 & $50-75$ & 0.0 & 850 & 21.24 & 40.21 & 24.29 & 0.00 & 0.33 & 0.67 & 0.83 & 5.00 \\
\hline 09 & $50-75$ & 1.2 & 840 & 21.32 & 39.76 & 25.04 & 0.00 & 0.42 & 0.58 & 14.17 & 16.67 \\
\hline 10 & $25-50$ & 0.3 & 720 & 21.09 & 39.57 & 24.69 & 0.33 & 0.08 & 0.58 & 15.83 & 15.00 \\
\hline 11 & $75-100$ & 3.9 & 900 & 21.37 & 39.76 & 24.56 & 0.75 & 0.00 & 0.25 & 13.33 & 25.00 \\
\hline 12 & $75-100$ & 1.8 & 940 & 20.76 & 40.26 & 24.19 & 0.50 & 0.33 & 0.17 & 14.17 & 50.83 \\
\hline 13 & $0-25$ & 2.4 & 680 & 21.41 & 39.78 & 24.03 & 0.00 & 0.50 & 0.42 & 0.45 & 0.00 \\
\hline 14 & $25-50$ & 5.4 & 930 & 21.18 & 40.27 & 24.40 & 0.00 & 0.25 & 0.75 & 6.67 & 2.50 \\
\hline 15 & $50-75$ & 1.3 & 960 & 21.51 & 39.51 & 24.49 & 0.33 & 0.42 & 0.25 & 8.75 & 55.00 \\
\hline 16 & $0-25$ & 10.0 & 880 & 21.32 & 39.47 & 24.84 & 0.00 & 0.33 & 0.58 & 15.00 & 8.33 \\
\hline 17 & $75-100$ & 9.2 & 590 & 21.42 & 39.76 & 24.33 & 0.50 & 0.33 & 0.17 & 14.17 & 15.00 \\
\hline 18 & $75-100$ & 1.6 & 930 & 21.25 & 40.18 & 24.27 & 0.60 & 0.00 & 0.40 & 4.00 & 48.00 \\
\hline 19 & $0-25$ & 13.0 & 910 & 21.34 & 39.82 & 23.96 & 0.08 & 0.83 & 0.08 & 8.75 & 0.83 \\
\hline 20 & $0-25$ & 2.0 & 810 & 21.36 & 39.95 & 24.78 & 0.00 & 0.20 & 0.80 & 0.00 & 0.00 \\
\hline
\end{tabular}


Table S4. Principal Component scores for each sampling site, in Pindos mountains area, taking into consideration the woody vegetation cover, grazing intensity, elevation, topographical wetness index, the proportion of dry leaves, bare ground and grass substrate, and the woody vegetation cover of more than $3 \mathrm{~m}$ and less than $3 \mathrm{~m}$ at a microhabitat scale.

\begin{tabular}{|c|c|c|c|c|c|c|c|c|c|}
\hline Site & PC 1 & PC 2 & PC 3 & PC 4 & PC 5 & PC 6 & PC 7 & PC 8 & PC 9 \\
\hline 01 & 0.78 & -0.94 & 2.89 & -0.73 & 1.46 & -0.36 & 0.43 & -0.16 & -0.01 \\
\hline 02 & -1.21 & 0.33 & 1.42 & 0.73 & -0.85 & 0.88 & -0.60 & -0.08 & 0.07 \\
\hline 03 & -1.61 & 0.49 & -0.07 & 0.85 & -1.09 & 0.01 & 0.41 & 0.45 & -0.04 \\
\hline 04 & -0.76 & -0.97 & -1.02 & 0.82 & -0.30 & -0.23 & 0.88 & -0.38 & -0.03 \\
\hline 05 & 2.09 & 1.44 & -0.60 & 0.15 & -1.04 & -0.43 & 0.42 & -0.36 & -0.03 \\
\hline 06 & 1.31 & 1.41 & 1.23 & 1.62 & -0.23 & -0.52 & -0.55 & -0.47 & 0.01 \\
\hline 07 & -0.58 & 0.99 & -1.08 & -0.88 & 0.88 & -0.67 & -0.25 & -0.06 & 0.07 \\
\hline 08 & -1.03 & -1.23 & -1.61 & -0.61 & 0.07 & -0.22 & 0.66 & -0.23 & 0.01 \\
\hline 09 & -0.64 & -1.35 & 0.28 & 0.61 & 0.16 & 1.46 & 0.94 & -0.22 & 0.01 \\
\hline 10 & 0.02 & -1.96 & 0.95 & -0.83 & -0.08 & 0.24 & -0.81 & -0.45 & 0.01 \\
\hline 11 & 2.71 & -0.58 & 0.49 & 0.12 & -0.67 & -0.81 & -0.04 & -0.25 & -0.03 \\
\hline 12 & 2.78 & 0.40 & -0.99 & -0.06 & 0.84 & 0.76 & 0.01 & -0.06 & -0.02 \\
\hline 13 & -2.22 & 0.42 & -1.04 & -1.94 & -0.22 & 0.34 & -0.77 & -0.36 & -0.13 \\
\hline 14 & -1.43 & -0.77 & -0.46 & 0.46 & 0.69 & -1.10 & 0.29 & 0.25 & 0.05 \\
\hline 15 & 1.74 & 0.15 & -1.33 & 0.79 & 0.65 & 1.44 & -0.42 & 0.31 & 0.02 \\
\hline 16 & -1.69 & 0.12 & 1.37 & 1.11 & 0.59 & -0.17 & -0.18 & 0.65 & -0.14 \\
\hline 17 & 0.97 & 1.02 & 1.50 & -2.41 & -0.97 & 0.34 & 0.63 & 0.63 & 0.04 \\
\hline 18 & 2.82 & -1.08 & -1.28 & -0.04 & -0.06 & -0.57 & -0.50 & 0.71 & 0.00 \\
\hline 19 & -1.79 & 3.98 & -0.27 & 0.03 & 0.67 & -0.05 & 0.10 & -0.10 & 0.04 \\
\hline 20 & -2.25 & -1.88 & -0.38 & 0.22 & -0.51 & -0.34 & -0.64 & 0.17 & 0.08 \\
\hline
\end{tabular}

